\title{
A Multivariate Fuzzy Clustering-Based Data Imputation for Adaptive Misbehavior Detection in Cooperative Intelligent Transportation Systems
}

\begin{abstract}
AUTHOR
Sultan Ahmed Almalki,
Computer Science Depat

Department University of Idaho Moscow, US A

ndals.uidaho.edu

Frederick Sheldon,

Computer Science Department University of Idaho Moscow, USA

\section{Abstract}

This work proposes a local-global fuzzy-clustering feature extraction scheme for detecting False Data Injection Attacks (FDIA). In this scheme, the data undergo several pre-processing steps including missing values imputation based on the local and global fuzzy-clustering correlation approach. There are four main components of the proposed method: i) data acquisition, ii) standardization, iii) normalization, and iv) imputation. To evaluate the performance of this scheme, the NGSIM dataset (described below) was used. This dataset contains data acquired from the environment using a set of sensors that collect data from the neighboring vehicles. The results show that the accuracy of models trained using said features extracted by the proposed scheme was higher than those proposed by the related studies. This indicates that the local-global fuzzy clustering data imputation approach proposed by this study can estimate the missing values better than existing techniques based on an exhaustive literature review.
\end{abstract}

\section{Proposed Model}

Pre- processing and Features Extraction

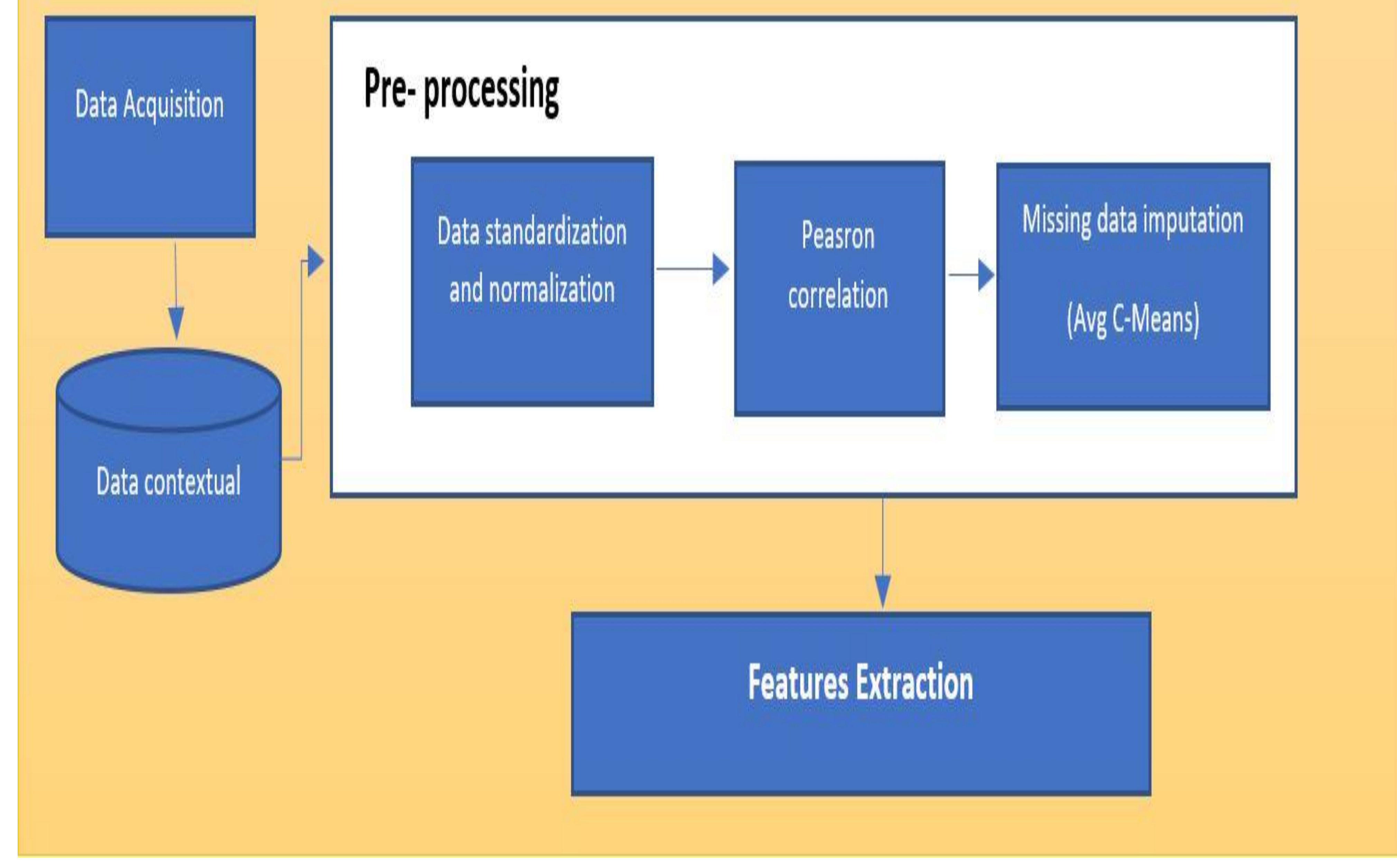

\section{Results}
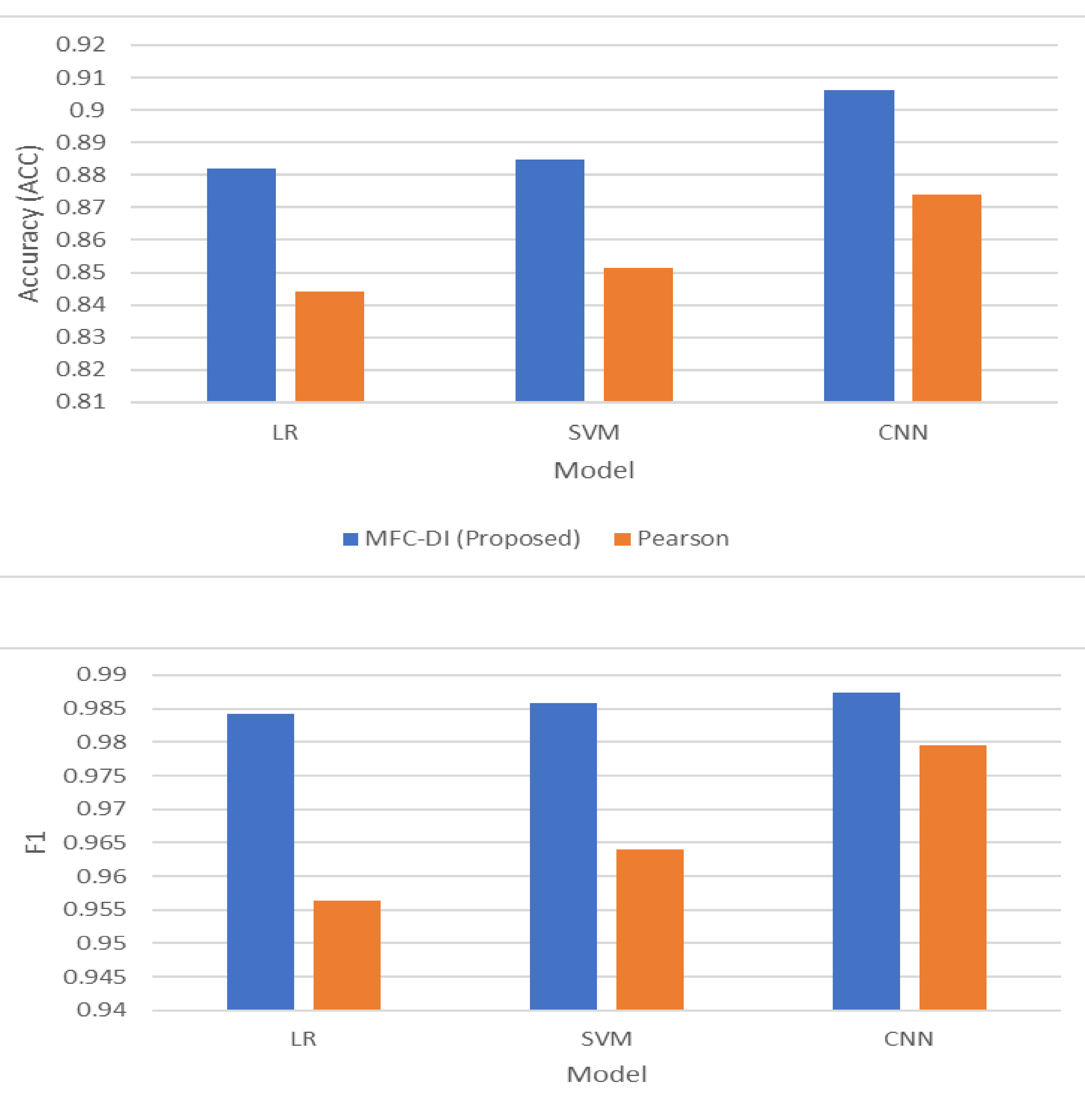

- MFC-DI (Proposed) - - Pearson

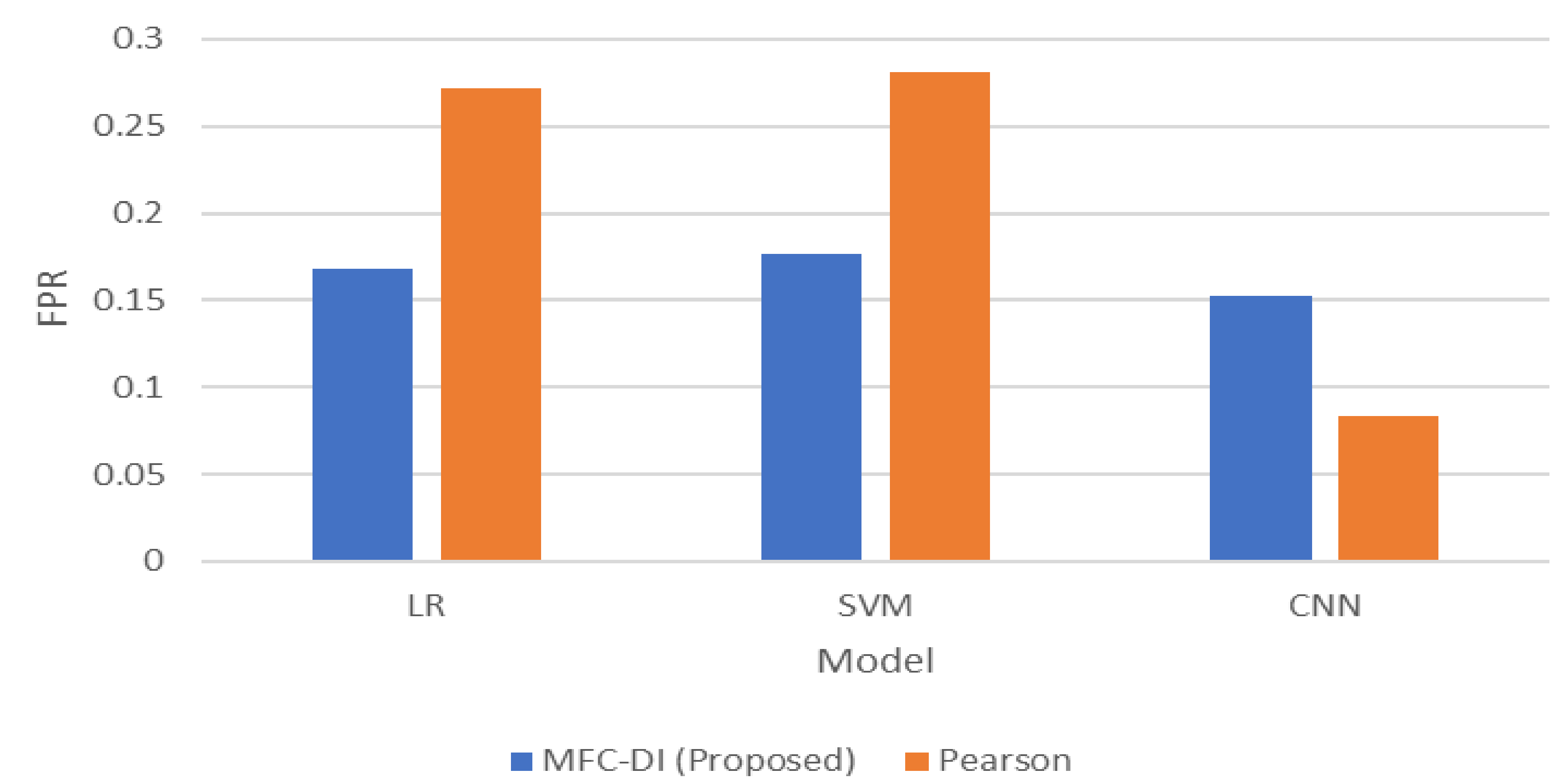

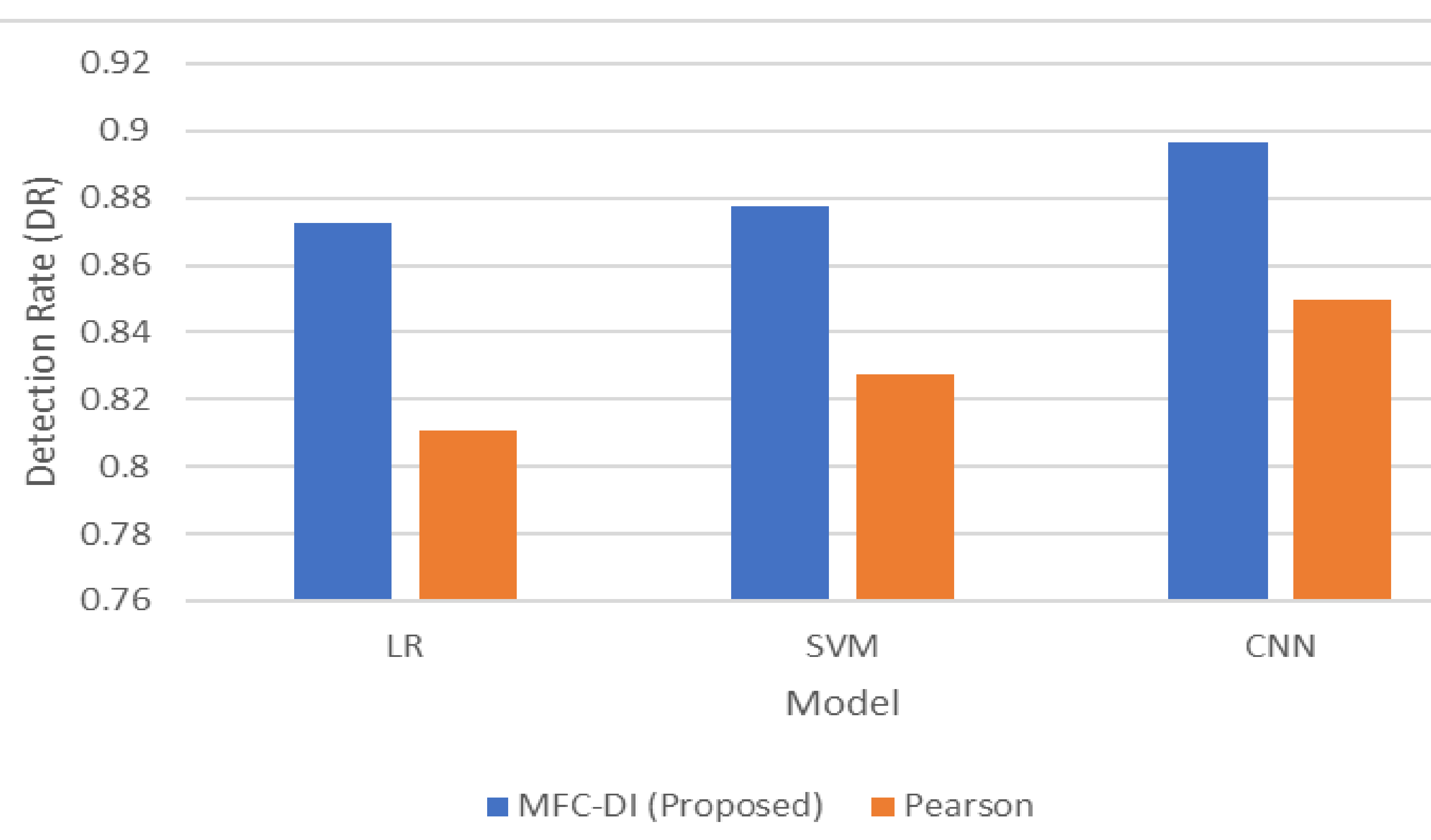

\section{Dataset}

The dataset in this research will be the Next Generation Simulation (NGSIM) Vehicle Trajectories Dataset. It is an open source and publicly available data set with a collection of real-world vehicles' trajectories collected by smart vehicles and consist of different patterns representing different drive situations and driver behavior. Particularly, NGSIM was built by collecting data from vehicles moving on a road section with $500 \mathrm{~m}$ long and seven lane highway. For each vehicle, the data is collected (recorded) for 45 minutes using 16 sensors .

Conclusion: Herein a new model, the Multivariate Fuzzy Clusteringbased Data Imputation (MFC-DI), is proposed. Our MFC-DI is a technique that approximates the missing values in the data acquired during the early phases of the new model's formation when the misbehaviour detection models in the cITS re-adjust to the environmental and contextual change. One caveat for adopting the multivariate approach is the computational overhead, especially for online models that adapt to the environmental and topological changes in the cITSs. This issue will be addressed in our future publication. The technique incorporates the Pearson correlation into the estimation of clusters membership weights. The cluster membership was used to approximate the missing values in data points based on the used to approximate the missing values in data points based on the
counterpart values in the other data points that share the same proximity from the clusters. In such a way the missing data are determined by the multi-variate attributes rather than univariate attributes used by conventional imputation techniques. The results show that the proposed MFC-DI outperformed those that have been obtained in the past using conventional Pearson correlation. We expect this will help to improve the adaptive and online cITS models and increases the reliability of the data exchanged between the vehicles on the road section.

\section{Universityof Idaho}

